\title{
Discours
}

Revue de linguistique, psycholinguistique et

informatique. A journal of linguistics, psycholinguistics

and computational linguistics

$6 \mid 2010$

Le fonctionnement en discours des énoncés averbaux autonomes

\section{Diverses fonctions discursives d'une phrase averbale en japonais formée par la séquence < proposition subordonnée + syntagme nominal >}

\section{Makoto Kaneko}

\section{(2) OpenEdition}

\section{Journals}

Édition électronique

URL : http://journals.openedition.org/discours/7768

DOI : $10.4000 /$ discours. 7768

ISSN : 1963-1723

Éditeur :

Laboratoire LATTICE, Presses universitaires de Caen

Référence électronique

Makoto Kaneko, « Diverses fonctions discursives d'une phrase averbale en japonais formée par la séquence < proposition subordonnée + syntagme nominal > », Discours [En ligne], 6 | 2010, mis en ligne le 29 septembre 2010, consulté le 19 avril 2019. URL : http://journals.openedition.org/ discours/7768 ; DOI : 10.4000/discours.7768

Ce document a été généré automatiquement le 19 avril 2019. 


\section{Diverses fonctions discursives d'une phrase averbale en japonais formée par la séquence < proposition subordonnée + syntagme nominal >}

Makoto Kaneko

\section{Introduction}

Cette étude a pour but d'examiner les diverses fonctions discursives remplies par un type de phrase averbale en japonais. Je commencerai par présenter certaines particularités de la syntaxe du japonais, qui sont pertinentes pour la discussion qui suit. L'ordre par défaut est sujet-objet-verbe, comme dans [1a]. Ne disposant pas de pronom relatif, le japonais contemporain forme une construction relative en mettant simplement le syntagme verbal (désormais $\mathrm{SV}^{1}$ ) devant le syntagme nominal (désormais $\mathrm{SN}$ ) servant de tête à la relative, comme dans $[1 \mathrm{~b}]^{2}$ :

$[1 \mathrm{a}]^{3}$

[suist Seko sensyu]-ga [obict $40 \mathrm{~km}$ titen]-o [nridicat kaityooni hasiru].

Seko coureur-Nom $40 \mathrm{~km}$ point-Acc en bonne forme courir

'Seko court avec entrain vers la borne des $40 \mathrm{~km}$.'

$[16]^{4}$

[relative kaityooni hasiru] [sN Seko-sensyu]-wa syoori-o kakusinsi-tei-ta en bonne forme courir Seko coureur-Top victoire-Acc sûr-Prog-Pst 'Seko, qui courait avec entrain, était sûr de sa victoire.' 
Il est maintenant à remarquer que certaines phrases, comme [2a], ne consistent qu'en une séquence $<\mathrm{SV}+\mathrm{SN}>$ et prennent la même forme que la construction relative dans [1b]. Il est possible d'y ajouter une copule qui sert de prédicat principal, comme dans [2b]. Considérant la phrase averbale «comme une phrase dont la proposition principale ne contient aucun verbe conjugué » (selon la définition proposée par Guillemin-Flescher, 2004 : 139), la séquence $<\mathrm{SV}+\mathrm{SN}>$ dans [2a] est analysée comme une phrase averbale :

[2a]

\author{
[sv kaityooni hasiru] [sN Seko-sensyu] ! \\ avec entrain courir Seko coureur \\ 'Seko qui court avec entrain !' [énoncé dans une émission sportive] \\ (Tsubomoto, $2000: 259$ )
}

$[2 b]^{5}$

[sv aikawarazu kayui tokoro-ni te-ga todoku] [sN Nakamura

comme toujours démanger endroit-Loc main-Nom arriver Nakamura

Syunsuke] dat-ta.

Syunsuke Cop-Pst

'On a vu Nakamura Syunsuke qui prêtait comme toujours une attention minutieuse aux petits détails.' (Asahi Sinbun 'Journal Quotidien Asahi', abrégé désormais en JQA, 28/08/2009)

Tsubomoto (2000: 256) fait remarquer que la phrase averbale en $<\mathrm{SV}+\mathrm{SN}>$, comme dans [1b], dénote un événement directement perçu et spatio-temporellement ancré dans la situation de perception, et la compare, dans cette optique, avec la séquence < SN qui SV >, appelée 'pseudo-relative' ${ }^{\prime}$ en français, comme dans [3a]. En effet, de même que la phrase averbale en $<\mathrm{SV}+\mathrm{SN}>\mathrm{du}$ japonais peut être accompagnée d'une copule comme dans $[2 \mathrm{~b}]$, celle en $<\mathrm{SN}$ qui $\mathrm{SV}>$ du français peut être introduite par un présentatif, comme voilà, c'est, il y a, dans [3b] :

[3a] Le lavabo qui déborde ! (Lefeuvre, $1999: 287$ )

[3b] \{Voilà / C'est / Il y a\} Le lavabo qui déborde.

Un examen minutieux des exemples attestés montre toutefois qu'à côté du type déictique de la phrase averbale, comme dans [2a], qui dénote un événement directement perçu et peut être mis en parallèle avec la pseudo-relative en $<$ SN qui SV $>$ du français, il existe d'autres types qui ne s'accordent pas avec une telle caractérisation : ainsi, dans [4], le SV dénote un état qui dure depuis longtemps, et qui n'est pas ancré dans le moment de la perception :

[4] 
[au début du synospis du film Le Fabuleux destin d'Amélie Poulain, trouvé dans la rubrique des programmes de télévision]

[sv Paris-no Montmartre-ni sumi café-de hataraku 22 sai-no]

Paris-Gen Montmartre-Loc habiter café-Loc travailler 22 ans-Gen

[sN Amélie]. Osanai-koro-kara utikina kanozyo-wa [...]

Amélie. enfance - depuis timide elle -Top

'Amélie, qui a 22 ans, habite à Montmartre, à Paris, et travaille dans un café.

Cette jeune fille, timide depuis son enfance, [...].' (JQA, 04/09/2003)

Presque aucune étude n'a été menée à ma connaissance sur ces emplois non déictiques de la phrase averbale. La présente étude précisera les particularités de ces emplois, en se basant sur des exemples tirés d'un journal quotidien, et en profitant aussi des études antérieures sur les phrases averbales dans d'autres langues, comme celle du français dans [5]:

[5] Sur le papier pourtant, personne ne conteste la qualité du travail effectué à Strasbourg, depuis près de dix ans, par l'ancien Premier ministre. Toujours présent, toujours actif, toujours prêt à régler un dossier ou à animer une mission! Sur la question de la future Constitution européenne, Rocard est également moins isolé au PSF qu'on veut bien le dire. (Le Nouvel Observateur, 30/10/2003 ; Delorme, $2004: 351$ )

La discussion sera menée dans l'ordre suivant : je passerai d'abord en revue les principaux travaux antérieurs sur la phrase averbale en $<\mathrm{SV}+\mathrm{SN}>\mathrm{du}$ japonais, qui caractérisent le type [2a] au moyen de trois valeurs, et observerai aussi des contre-exemples à leurs analyses, comme [4] (section 1); afin de mieux comprendre ces contre-exemples, j'envisagerai ensuite les analyses antérieures des phrases averbales dans d'autres langues, comme celle du français dans [5] (section 2) ; je proposerai enfin de classifier les emplois de la phrase averbale du japonais en quatre types, et clarifierai les caractéristiques de chaque type (section 3) ;la section 4 récapitulera les résultats de cette étude.

\section{Travaux antérieurs sur la phrase averbale en < SV $+\mathrm{SN}>$ du japonais et la pseudo-relative en $<\mathrm{SN}$ qui SV $>$ du français}

\subsection{Kantai, jugement thétique et pseudo-relative}

7 La phrase averbale en $<\mathrm{SV}+\mathrm{SN}>$ est attestée depuis le japonais classique, notamment dans les poèmes lyriques, comme dans [6a] :

[6a]

[sv Mikasa-no yama -ni ide-si] [sN tuki] kamo.

Mikasa-Gen montagne-Loc apparaître-Pst lune particule emphatique

'La lune qui est apparue sur le Mont Mikasa!' (partie d'un poème composé au

VIII॰ siècle par Abe no Nakamaro)

[6b] 
[ss kono tuki]-wa [sv Mikasa-no yama-ni ide-ki]
cette lune-Top Mikasa-Gen montagne-Loc apparaître-Pst
'Cette lune est apparue sur le Mont Mikasa.' (Yamada, 1936: 938)

8 Cette construction est fréquemment discutée par les chercheurs en linguistique traditionnelle japonaise. Parmi eux, Yamada (1936) la baptise Kantai (phrase expressive), et l'oppose aux phrases comme [6b], qu'il appelle Zyuttai (phrase prédicative). Selon cet auteur, le Zyuttai correspond à la 'proposition' au sens logique et associe le Syukaku (correspondant au Sujet ou à l'argument en termes logiques) au Zyukkaku (correspondant au Prédicat) (Yamada, 1936: 945-946). Le Kantai serait émis comme une réaction spontanée, juste après la perception, sans que le locuteur reconnaisse la dichotomie : il est souvent accompagné d'affectivité, d'où son appelation Kantai (phrase expressive) (Yamada, 1936: 936). Du point de vue syntaxique, Yamada analyse le Kantai comme une relative suivie par la tête, compte tenu du fait que, dans le japonais classique qui dispose de la distinction morphologique entre les formes adnominale et conclusive du verbe, le prédicat du Kantai dans [6a] prend la forme adnominale, et celui du Zyuttai dans [6b], la forme conclusive. Le contraste entre Kantai et Zyuttai peut donc être mis en parallèle avec celui entre le jugement thétique et le jugement catégorique ${ }^{7}$, que Kuroda (1992) définit dans les termes suivants :

Categorical judgment « consists of two distinct cognitive acts, one recognition of the [logical] Subject and another the act of acknowledging or disavowing a [logical] Predicate of a Subject ", and " associating a Subject with a Predicate ». (Kuroda, 1992: 21)

Thetic judgment is "a unitary cognitive act» and "a direct response to the perceptual cognition of an actual situation ». (Kuroda, 1992:22)

\subsection{Caractéristiques partagées par le Kantai en japonais et la pseudo-relative averbale en français}

9 Sasse (1987) et Furukawa (1996), entre autres, soutiennent que la pseudo-relative du type [7a] incarne aussi un jugement thétique. En effet, les deux constructions partagent trois caractéristiques - déicticité, bloc sémantique, information non interactionnelle - qui s'accordent avec les définitions du jugement thétique chez Kuroda (1992), et qui permettent par ailleurs de distinguer les pseudo-relatives des relatives restrictives ou appositives, illustrées par [7b], malgré leurs ressemblances apparentes :

[7a] Tiens ! Le facteur qui passe ! [pseudo-relative] (Furukawa, 1996: 64)

[7b] Tu connais le facteur qui passe? [relative restrictive]

\subsubsection{Déicticité}

Selon Kuroda (1992: 22), le jugement thétique est « une réponse directe à la cognition perceptuelle de la situation actuelle ». Du coup, l'événement dénoté doit en principe être perceptible et ancré dans la situation de perception. Onoe (2001:270) caractérise de fait la phrase averbale en $<\mathrm{SV}+\mathrm{SN}>\mathrm{du}$ japonais comme une «expression attachée à la situation de perception (genba-mityaku-gata-no hyoogen)».Cette nature déictique est partagée selon Furukawa (1996 : 143) par la pseudo-relative averbale en français, dont « le point d'ancrage [doit être] constitué par le lieu d'énonciation ». De même, Cadiot (1976) 
soutient, en l'appelant " relative déictique », que cette construction implique " une saisie directe de l'objet référé, une saisie 'hic et nunc' »".

11 Afin de rendre compte de cette déicticité, Erteschik-Shir (1997) ${ }^{9}$, entre autres, soutient que les phrases thétiques disposent d'un argument spatio-temporel implicite. Dans la même veine, Lefeuvre (1999: 287) avance que la phrase averbale dans [8a] correspond au rhème dont le thème implicite, de nature spatio-temporelle, est récupéré par la situation d'énonciation, comme le schématise [8b]:

[8a] Le lavabo qui déborde ! (Lefeuvre, 1999 : 287)

[8b] $\left[_{\text {thème }} \text { (ici et maintenant) }\right]_{\text {rhème }}$ le lavabo qui déborde]

12 Une telle déicticité est d'abord identifiée par une restriction sur les prédicats compatibles. Les deux constructions n'admettent pas les prédicats qui ne sont pas spatiotemporellement délimités, comme les prédicats permanents dans [9a et b], et les prédicats habituels dans [9c] :

[9a] ${ }^{*}$ Le facteur qui est grand ! (vs [7a])

[9b]

??[se- ga takai] Hanako ! (vs [2a])

taille-Nom grand Hanako

'Hanako qui est grande !' (Tsubomoto, 1992 : 572)

[9c]

[(??itumo) Diru-no apaato-no soto -o aruiteiru] Faagasu.

toujours Dil-Gen appartement-Gen dehors-Acc marcher Fergus

'Fergus qui marche (??toujours) autour de l'appartement de Dil.'

(Tsubomoto, $2000: 253$ )

13 Ensuite, les deux constructions ne sont possibles en principe qu'au présent ${ }^{10}$, comme le montre l'acceptabilité dégradée de [11a et $\mathrm{b}]$ dont le prédicat est mis au passé :

[10a] ??Le facteur qui passait ${ }^{11}$ ! (vs[7a]) (Furukawa, $\left.1996: 143\right)$

$[10 \mathrm{~b}]$

??[izen-wa marathon-o siteita] Seko !

autrefois-Top marathon-Acc faisait] Seko (vs [1b])

'Seko qui faisait du marathon autrefois !' (Tsubomoto, 1992 : 572)

14 Toutefois, le présent ne renvoie pas toujours au moment de l'énonciation, en français ainsi qu'en japonais. Outre les emplois visiblement non déictiques exprimant un fait universel, comme dans Deux et deux font quatre, ou une habitude, comme dans Je vais à l'école tous les jours, les deux langues ont un emploi appelé 'présent historique' qui renvoie à un moment du passé, comme dans Le 31 janvier 1907, le quotidien Le Matins'interroge. J'analyserai le présent historique comme mettant en œuvre le déplacement du centre déictique sur le passé. 


\subsubsection{Bloc sémantique} par le pronom de la troisième personne féminine, kanozyo, dans [12a], la même séquence analysée comme une phrase averbale est reprise par le démonstratif sore 'cela', ou par un nom événementiel, sono yoosu 'cette situation', dans [12b] :

[12a]

[Odoroki kanasimu] Aida-wa itumo-nimo-masite utukusikat-ta. \{*sore / s'étonner se lamenter Aida-Top plus que d'ordinaire beau-Pst cela kanozyo \}-o Amneris-wa aratamete nikun-da.

elle \} Acc Amneris-Top à nouveau haïr-Pst

'Aida, qui était étonnée et se lamentait, était plus belle que d'ordinaire. Amneris l'a haïe à nouveau.'

$[12 b]$

[Odoroki kanasimu] Aida. \{sore-o / sono yoosu-o \} mi-ta Amneriss'étonner se lamenter Aida cela-Acc/cette situation-Acc voir-Pst Amneriswa kokoro hisokani yorokon-da.

Top cœur au fond se réjouir-Pst

'Aida qui s'étonne et se lamente. Amneris, qui avait vu \{cela / cette situation\}, s'est réjouie au fond de son ccurr.' (adapté de Tsubomoto, $2000: 261$ )

En second lieu, Léard (1992: 131) observe que les pseudo-relatives "valent seulement pour le sujet, [et] sont toujours introduites par qui». Cette contrainte est illustrée par le contraste entre [13a] où le $\mathrm{SN}$ correspond au sujet et [13b] où le $\mathrm{SN}$ correspond à l'objet. Le même contraste est observé pour la séquence $<\mathrm{SV}+\mathrm{SN}>$ en japonais, comme dans [14a et b] :

[13a] Tiens ! Le facteur qui passe ! (Furukawa, 1996:64)

[13b] ??Oh ! Le facteur que Pierre renverse ! (Furukawa, $1996: 64)$

[14a $]^{15}$ 
[Takanohana-o osu] Akebono!

Takanohana-Acc pousser Akebono

'Akebono qui pousse Takanohana!'

$[14 \mathrm{~b}]$

??[Akebono-ga osu] Takanohana!

Akebono-Nom pousser Takanohana

'Takanoha qu'Akebono pousse!' (Tsubomoto, $2000: 252$ )

19 Léard (1992: 131) rend compte de cette restriction en affirmant qu'« en l'absence de hiérarchie dans l'information apportée par les éléments, tous nouveaux, il n'y a pas de raison de bouleverser l'ordre des constituants ». Autrement dit, si le SN correspond à un autre élément que le sujet, son référent est nécessairement topicalisé et la phrase entière s'avère être un jugement catégorique.

\subsubsection{Information non interactionnelle}

Yamada (1936) fait remarquer que son Kantai transmet une valeur affective, qui est renforcée dans [6a] par la particule emphatique à la fin de la phrase. Dans la même veine, Sandfeld (1965 : 156) parle, à propos des phrases comme [15], de «valeur fortement affective de la phrase (étonnement, regret, vive opposition, etc.) » :

[15] Ah! Mon dieu! dit-il, Monsieur Michel qui est mort! (Sandfeld, 1965 : 155)

Il est à noter que Beyssade et Marandin (2006) caractérisent l'exclamation ou l'étonnement par leur nature non interactionnelle : à la différence des autres types de phrases (i.e. déclarative, interrogative et impérative), la phrase exclamative ne requiert que l'implication du locuteur et ne fait aucune demande à l'interlocuteur. Marandin (2005), quant à lui, envisage l'état informationnel des participants au dialogue, et le divise en deux parties, publique et privée, comme le schématise [16]. Cet auteur distingue par ailleurs deux types de fonds (ground en anglais) : fonds dans la partie publique, partagé en commun par les interlocuteurs (common ground) et fonds en arrière-plan dans la partie privée, qui correspond à «la description du but du locuteur et la description de son savoir » (Marandin, $2005:$ 53) :

[16] [État Informationnel [Public (...fonds partagé...)] [Privé (...fonds en arrière-plan)]] (adapté de Marandin, $2005:$ 51-53)

Dans cette optique, le Kantai du japonais et la pseudo-relative du français ne visent pas à faire partager l'information par les interlocuteurs. L'étonnement est dû au fait que l'information ne s'accorde pas aux connaissances du fonds privé du locuteur.

En japonais, au moins deux critères permettent de comprendre qu'il s'agit d'une information du fonds privé. D'abord, Kuroda (1979: 235-236) note que « des adjectifs comme chaud, qui expriment des sensations ou des émotions ressenties par le sujet, sont épistémologiquement très différents des autres adjectifs", comme grand, et que le japonais distingue morphologiquement une sensation ou émotion intérieure du sujet de sa manifestation extérieure. Ainsi, l'adjectif atui 'chaud', qui décrit une sensation subjective, n'est en principe compatible qu'avec le sujet à la première personne (ex. [17a] 
vs [17b]). Le sujet à la troisième personne exige une forme différente indiquant une manifestation extérieure, atugaru 'montrer sa chaleur' :

[17a]

watasi-wa atui.

moi-Top chaud

'J'ai chaud.' (Kuroda, $1979: 235$ )

$[17 \mathrm{~b}]$

*Jean-wa atui. [dans le style rapporté]

Jean-Top chaud

'Jean a chaud.'

24 Kuroda (1979 : 242) fait de plus remarquer que « la restriction sur le sujet des adjectifs de sensation peut être levée lorsqu'une phrase qui contient un tel adjectif est incluse » dans un style non rapporté (style indirect libre). Pour revenir à la phrase averbale, dans [12b] cité plus haut, le verbe exprimant l'état intérieur, odoroku 's'étonner', est associé au sujet de troisième personne; dans [18], une onomatopée, gan 'gang', transmet une sensation auditive des voyageurs.

Le deuxième critère qui permet d'identifier une information non interactionnelle est aussi observé dans [18]. Dans cet exemple, le tiret qui suit la phrase averbale clarifie le fait que le plan énonciatif des deux premières phrases est distinct de celui de la partie suivante, qui rapporte l'accident objectivement, en précisant la date, le modèle et le trajet du train, ainsi que le nombre des voyageurs :

[18]

[au début d'un article qui rapporte le déraillement accidentel d'un train]

gan toyuu syoogeki. [sv sikiisi-no ue- o tobihaneru-yooni hasiru].

Gang ! citation choc gravier-Gen dessus-Acc sautiller comme si courir]

[sN syaryoo] - 19 niti, Kyoto-kara Osaka-e mukau Keihan-densya-no

train 19 jour, Kyoto-de Osaka-à aller Keihan-train-Gen

tokkyuu-densya-de oki-ta dassen -ziko -wa, yaku 300

express-train-Loc avoir lieu-Pst déraillement-accident-Top, environ 300

nin -no zyookyaku-o odoroki-to kyoofu-ni otosiire-ta.

personne-Gen voyageur-Acc Surprise-et peur-Dat plonger-Pst

" "Gang ! » un choc. Le train qui roule sur le gravier en sautillant. - le

déraillement accidentel, le 19 août, d'un train express de la société Keihan qui allait de Kyoto à Osaka, a plongé environ 300 voyageurs dans la surprise et la peur.' (JQA, 20/08/2003)

Outre l'information non interactionnelle, cet exemple présente les deux autres caractéristiques du Kantai : i) l'accident a lieu au passé, comme l'indique la morphologie du passé dans la $3^{\mathrm{e}}$ phrase, tandis que le SV de la phrase averbale est mis au présent, et se trouve ainsi ancré dans le moment de la perception; ii) le SN, syaryoo 'train', correspond au sujet du prédicat, hasiru 'courir'. Par ailleurs, le topique de la phrase suivante, dassenziko 'déraillement accidentel', reprend l'événement dénoté par la séquence < SV + SN > entière, et non pas le référent du SN seul. La phrase averbale présente donc l'événement comme formant un bloc sémantique. 


\subsection{Contre-exemples} qui ne s'accordent pas avec les trois caractéristiques du Kantai. Dans [19], la marque d'évidentialité toyuu 'dit-on', dans le SV, indique qu'il ne s'agit pas d'une perception directe. Ensuite, ce n'est pas l'ensemble de la phrase averbale, mais le SN seul, qui est repris comme topique implicite dans la phrase suivante. De plus, la contrainte grammaticale sur le SN est levée, et le SN correspond à l'objet du SV, motu 'posséder'. Enfin, cet exemple vise à faire partager aux lecteurs l'information transmise. Il ne s'agit donc pas d'une information non interactionnelle :

[19]

[au début d'un article sur les caméras vidéo]

[sv imaya ippan setai-no 4 wari-ga motu toyuu] [snvideo-camera]. aujourd'hui standard famille-Gen $40 \%$-Nom posséder on dit vidéo caméra

Kodomo-ya mago-no tanzyoo-ga kikkake-de kau hito

Enfant- et petit-enfant-Gen naissance-Nom occasion-Loc acheter personne

-ga ooi-ndatte.

-Nom nombreux-il est dit que

'Les caméras vidéo que, dit-on, $40 \%$ des familles ordinaires possèdent.

Il est dit que beaucoup de gens en achètent une à l'occasion de la naissance de leur enfant ou de leur petit-enfant.' (JQA, 06/09/2009)

Il est à remarquer que les contre-exemples [4] et [19] apparaissent tous les deux au début d'un texte et servent à introduire un topique discursif. Ces observations suggèrent que l'analyse appropriée des phrases averbales du japonais exige de prendre en compte leur place dans le texte et leurs fonctions discursives. Afin de mieux faire connaître ces facteurs, j'aborderai, dans la section suivante, certains travaux antérieurs sur la phrase averbale dans d'autres langues.

\section{Travaux antérieurs sur les fonctions discursives de la phrase averbale dans d'autres langues}

Beaucoup de travaux récents examinent les fonctions discursives de la phrase averbale dans les différents genres (fiction, presse, didascalie, entretien, débat oral, etc.) en français, en anglais ou en allemand, langues où la phrase averbale n'est pas, de même qu'en japonais, une construction dominante. Dans cette section, en vue de leur application à l'analyse de la phrase averbale du japonais, j'examinerai certains travaux antérieurs qui abordent trois aspects de la phrase averbale : i) plan énonciatif; ii) rôles discursifs en fonction de la place dans le texte ; iii) relations de discours.

\subsection{Introduction d'un nouveau plan énonciatif}

Il a été suggéré dans la section 1.2 que la phrase averbale en $<\mathrm{SV}+\mathrm{SN}>$ du japonais, ainsi que celle en $<\mathrm{SN}$ qui $\mathrm{SV}>$ du français, transmet une information non interactionnelle. Examinant les emplois de la phrase averbale dans la fiction, Delorme (2004: 344) soutient qu'elle introduit « un point de vue nouveau dans le flux narratif, différent de celui de 
l'énonciateur primaire ». Ainsi, la phrase averbale soulignée dans [20] est décrite du point de vue d'un protagoniste référé par he:

[20] Something dripped on his shoulder. [...] He smelled it. Blood. (Delorme, $2004: 343)$

[21] A voice was echoing through the open double doors to the meeting hall. A deep, white-sounding voice. (K. Mitchelle, Deep Valley Malice; Delorme, $2004: 350)$

Delorme (2004: 350) fait par ailleurs remarquer que, dans [21], « a deep white-sounding voice n'est vraisemblablement pas mimétique des pensées du protagoniste [...] dans la mesure où le syntagme nominal [...] est trop écrit, trop descriptif pour être imputable à la pensée [...] du personnage en situation». Selon cet auteur, une telle "prise en charge plurielle » est due à l'absence "de marques explicites de repérage personnel, modal et temporel ", ce qui fait que «les prédicats concernés sont libres de s'associer à toute instance pertinente dans la situation de récit». Cette caractérisation devrait s'appliquer aussi à la phrase averbale du japonais, qui n'est pas accompagnée de la copule.

Selon Delorme (2004: 351), le transfert du point de vue est observé non seulement dans la fiction, mais aussi dans la presse. Ainsi, dans [22], «le prédicat adjectival semble introduire un point de vue en rupture avec celui de l'énonciateur " (ce qui est confirmé par le point d'exclamation), et la prise en charge est attribuée aux « collaborateurs de M. Rocard au conseil de l'Europe » :

[22] Sur le papier pourtant, personne ne conteste la qualité du travail effectué à Strasbourg, depuis près de dix ans, par l'ancien Premier ministre. Toujours présent, toujours actif, toujours prêt à régler un dossier ou à animer une mission! Sur la question de la future Constitution européenne, Rocard est également moins isolé au PSF qu'on veut bien le dire. (= [5])

D'autre part, selon Delorme et Lefeuvre (2004 : 286-287), le transfert suscité par la phrase averbale ne porte pas toujours sur la prise en charge de la perspective, mais aussi simplement sur le plan textuel. Ainsi, dans [23], la phrase averbale indique «le passage d'une vision objective du référent[ex. il se rassied, il a les yeux rivés sur le mur d'en face] à une vision plus subjective [i.e. prédicat psychologique, insensible aux émotions des témoins ]»:

[23] Puis, l'homme gris se rassied, les yeux rivés sur le mur d'en face. Insensible aux émotions des témoins. Mireille Mendès France : "ça m'a rappelé les caricatures qu'on faisait de mon beau-père ». (Le Nouvel Observateur; Delorme et Lefeuvre, 2004 : 283)

\subsection{Fonction discursive et place dans le texte}

Examinant la phrase averbale dans les didascalies, Krazen (2004:355) observe que sa fonction et sa portée dépendent de sa place dans le texte : i) au début d'une pièce, d'un acte ou d'une scène, elle sert à établir un tableau et étend sa portée « sur une portion plus ou moins longue (la pièce, un acte ou une scène entiers)»(Krazen, 2004: 360). Par exemple, dans [24], les première et deuxième phrases averbales établissent un cadre spatial et temporel qui reste valable dans l'acte entier; ii) au milieu du texte, elle participe « au processus dramatique » ou " au déroulement chronologique », et sa portée est moins longue, comme dans [25] : 
[24] Une chambre à coucher. La nuit. Une lampe sur une table. (Hugo, Hernan

i ; Krazen, $2004: 366$ )

[25] SOEUR ÉPINE, glaciale

Non, merci, pas ce fauteuil. (Étonnement d'Elvire et de Franklin. Un temps)

Vous avez forniqué tous les deux dans ce fauteuil! (La baby-sitter ; Krazen,

$2004: 368)$

En envisageant la phrase averbale dans les articles de presse, Lefeuvre (2004a) aussi reconnaît ces deux fonctions : elle sert soit i) à fournir des informations tout en étant pleinement rhématique, soit ii) à donner un commentaire prospectif ou rétrospectif tout en perdant sa densité rhématique. Le premier type, 'informatif', est utilisé notamment au début d'un paragraphe pour signaler l'existence d'un objet ou d'une situation. Le second type, 'commentaire', est illustré par [26a]. Cet exemple montre aussi que, même au milieu du texte, la portée de la phrase averbale prédicative peut dépasser une phrase: «les prédicats averbaux Insensé, incroyable et pourtant bien réel portent à la fois sur ce qui précède et sur ce qui suit » (Lefeuvre, 2004a : 193), comme l'indique la paraphrase dans [26b]. Ainsi, la phrase averbale « joue un rôle de pivot entre le contexte de gauche et le contexte de droite » (Delorme \& Lefeuvre, $2004: 281$ ) :

[26a] Le prévenu Dumas s'est comporté, mercredi à la barre, comme un sauvageon [...]. Insensé, incroyable et pourtant réel. Roland Dumas [...] a brisé le masque et sa propre statue d'homme de marbre. (Le Monde, 02/02/2001; Lefeuvre, 2004a : 193)

[26b] Ce qui est insensé, c'est aussi bien le fait que le prévenu Dumas se soit comporté comme un sauvageon que le fait qu'il ait brisé le masque et sa propre statue d'homme de marbre. (Lefeuvre, 2004a : 194)

\subsection{Relations de discours}

Lefeuvre (2004b) envisage la phrase averbale centrée sur la nominalisation, et examine l'interaction entre le type aspectuel du procès et la relation de discours qu'elle établit. Pour le classement des types aspectuels, cet auteur recourt à Vendler qui distingue i) état, illustré par être grand, ii) activité, dont le procès a une durée et n'a pas de borne finale, illustré par dormir, iii) accomplissement, dont le procès a à la fois une durée et une borne finale, illustré par construire une maison, iv) achèvement, dont le procès est ponctuel, illustré par arriver.

Quant aux relations de discours, elle distingue, avec Asher et Lascarides $(2003)^{16}$, i) Arrièreplan, où une proposition fournit une information sur la situation dans laquelle a lieu un événement dénoté par la proposition adjacente, comme dans [27a] ; ii) Élaboration, où une proposition fournit une information plus détaillée sur le fait dénoté par la proposition précédente, comme dans [27b] ; iii) Explication, où une proposition fournit la cause du fait dénoté par la proposition précédente, comme dans [27c] ; iv) Résultat, où une proposition fournit un effet apporté par le fait dénoté par la proposition précédente, comme dans [27d]; v) Contraste, qui présente des procès incompatibles, comme dans [27e]; vi) Narration, qui raconte une succession de procès, comme dans [28] ; vii) Continuation, qui raconte des procès qui ont lieu parallèlement, mais non pas successivement, comme dans $[29]^{17}$ :

[27a] Max a ouvert la porte. La chambre était bien sombre. [Arrière-plan] 
[27b] Max a pris un bon repas hier soir. Il a mangé beaucoup de saumon. [

Élaboration]

[27c] Max est tombé. Jean l'avait poussé. [Explication]

[27d] Jean a poussé Max. Max est tombé. [Résultat]

[27e] Jean aime le sport. Mais il déteste le foot. [Contraste]

[28a] Max est entré dans la chambre.

[28b] Il s'est assis. [Narration]

[29a] Le prof a demandé aux étudiants de chercher le chat.

[29b] Jean a cherché sous la table.

[29c] Marie a cherché au jardin. [Continuation (29b et c)]

\section{relation de Continuation entre elles, et une relation de Résultat avec les phrases} précédentes :

[32] Cette fois elle est en passe de se laisser fléchir, quand le juge Éric de Montgolfier lui fait savoir que, dans ce cas-là, il l'inculperait pour dénonciation calomnieuse. Fureur [état] au château où Johnny n'a que des amis. Et volonté [état] affichée de se débarrasser d'un magistrat peu coulant. (Marianne, 7-13/08/2003; Lefeuvre, 2004b : 316)

\subsection{Récapitulation}

41 Cette section a présenté certains des résultats obtenus par les études sur les phrases averbales en français et en anglais. On peut les récapituler ainsi : i) la phrase averbale dans la fiction, ainsi que dans la presse, sert à déplacer un point de vue ou un plan énonciatif; ii) dans les didascalies ainsi que dans la presse, la phrase averbale au début du texte apporte une information portant sur le texte entier, en introduisant un topique discursif ou un cadre spatio-temporel, tandis qu'au milieu du texte, elle présente un procès dynamique dont la portée est étroite, ou un commentaire prospectif ou rétrospectif dont la rhématicité est dégradée ; iii) les phrases averbales en nominalisation établissent diverses relations de discours avec les phrases précédentes et suivantes, ou entre elles, selon le type aspectuel du procès qu'elles dénotent. 


\section{Les quatre types de phrase averbale en $<\mathrm{SV}$ $+\mathrm{SN}>$ du japonais} présente les trois caractéristiques du Kantai : déicticité, bloc sémantique, information non interactionnelle. La section 2 a présenté trois facteurs pertinents pour envisager les fonctions discursives de la phrase averbale dans les autres langues: transfert du plan énonciatif; corrélation entre la place dans le texte et la portée informative ; interaction entre le type aspectuel du procès et la relation de discours. Dans cette section, en tenant compte de ces résultats, je proposerai de classifier les phrases averbales en $<\mathrm{SV}+\mathrm{SN}>\mathrm{du}$ japonais en quatre types.

\subsection{Type incarnant un Kantai}

Le premier type est illustré par [18] (voir section 1.2) et [33]. Il a été montré en section 1.2 que [18] présentait les trois caractéristiques du Kantai. Il en est de même pour [33]. D'abord, bien qu'il s'agisse d'un événement au passé, le SV est morphologiquement mis au présent, et indique l'ancrage dans le moment de la perception. Ensuite, le SN, hito 'personne', correspond au sujet des verbes, hakobidasu 'emporter' et orosu 'descendre', et le contenu des deux phrases averbales est repris dans la phrase suivante par l'expression fukkyuu 'reconstruction', qui dénote un procès plutôt que les référents des SN. Enfin, tandis que le texte commence par présenter objectivement le sinistre en précisant le lieu, la date et les dégâts, les phrases averbales introduisent un nouveau plan énonciatif dont la perspective n'est pas due seulement à l'auteur de l'article, mais aussi aux habitants et même aux lecteurs. Le tiret qui suit les deux phrases averbales indique le retour au plan énonciatif originel dont la prise en charge est exclusivement assumée par l'auteur :

[33]

[au début d'un article qui parle d'une région touchée par une trombe]

Mimasaka-si Aso-tiiki-o tatumaki-to mirareru toppuu

Mimasaka-ville Aso-secteur-Acc trombe-Comp considéré coup de vent

-ga osot-ta no-wa 7-gatu 19-niti. Zen-kai 2 setai

-Nom frapper-Pst Comp-Top juillet 19 jour. Totalement-détruit 2 maison

-0 fukumu 74 setai-ga hisaisi-ta. [sv Gareki-o hakobidasu] [sN hito].

-Acc inclure 74 maison-Nom subir-Pst décombre-Acc emporter personne

[sv Yane-kara kowareta kawara-o orosu] [ss hito] - 1 ka getu izyoo tati, toit-Abl cassé tuile-Acc descendre personne un mois plus de passer, zyuumin soodede fukkyuu-ni atat-tei-ta syuuraku-wa tous les habitants reconstruction-Dat s'occuper-Prog-Pst zone-Top ima sidukesa-ni tutumare -teiru.

maintenant calme-par enveloppé -Prog

'Le secteur Aso de la ville de Mimasaka a été frappé le 19 juillet par un coup de vent terrible considéré comme une trombe. Ce sinistre a endommagé 74 maisons, dont 2 ont été totalement détruites. Quelqu'un qui emporte des décombres. Quelqu'un (d'autre) qui descend des tuiles cassées du toit. - Un mois a passé depuis, et la zone que tous les habitants s'occupaient à reconstruire à ce moment-là est plongée maintenant dans le calme.' (JQA, 04/09/2009) 

mileu, comme dans [33]. Du point de vue aspectuel, du fait que ce type dénote un événement directement perçu, le prédicat doit avoir une certaine durée, et est donc en principe de type activité, comme hasiru 'courir' dans [18], ou accomplissement, comme hakobidasu 'emporter' et orosu 'descendre' dans [33]. Quant à la relation de discours, la phrase averbale dans [18] (qui rapporte une sensation visuelle) établit une relation de Continuation avec la phrase précédente (qui présente une sensation auditive par l'onomatopée), et une autre d'Explication avec la phrase suivante (qui permet aux lecteurs de comprendre que l'événement décrit est causé par le déraillement du train). D'autre part, dans [33], les phrases averbales décrivent la réparation des dégâts, tandis que la phrase précédente décrit la trombe qui en est la cause. La relation dont il s'agit est donc celle de Résultat. La relation entre les deux phrases averbales est identifiée comme Continuation.

\subsection{Type présentant une situation sur un nouveau plan énonciatif}

Le second type est illustré par [34a et b], qui est tiré du synopsis d'un feuilleton dans la rubrique des programmes de télévision. Dans chaque cas, la phrase averbale est mise à la tête du synopsis et présente une situation qui sert de base au développement de l'action dans l'épisode en question. Le SN dénote un des protagonistes, qui sont déjà (censés être) familiers des lecteurs :

[34a]

[au début du synopsis du $7^{\circ}$ épisode du feuilleton Black Jack ni yorosiku ${ }^{\circ} \mathrm{Dis}$ bonjour au Dr. Black Jack', qui décrit les activités des internes et des médecins dans un hôpital]

[sv umaretekita hutago-o zibun-no kodomo da to mitome-taku venir de naître jumeaux-Acc soi-Gen enfant Cop Comp admettre-vouloir -nai] [sN Hidekatu to Yosiko]. Eiziroo-wa sono zijzitu-ni odoroku ga, Neg Hidekatu et Yosiko. Eiziroo-Top ce fait-Dat s'étonner mais, kangofu-no Minagawa-wa mezurasii koto de-wa nai to hanasu. infirmière-Gen Minagawa-Top exceptionnel cas Cop-Top Neg Comp dire. 'Hidetake et Yosiko qui ne veulent pas admettre que les jumeaux qui viennent de naître sont leurs propres enfants. Eiziro s'étonne de ce fait tandis que Miyagawa, une infirmière, lui dit que ce n'est pas un cas exceptionnel.' (JQA, 23/05/2003)

[34b]

[au début du synopsis du $4^{\circ}$ épisode du même feuilleton]

[sv Miyamura-no syuzyutu-ga kikenna koto-o siri, betu byootoo-no Miyamura-Gen opération-Nom risqué fait-Acc savoir, différent section-Gen Kita-ni syuzyutu-o tanon-da] [sN Eiziro]. Sikasi, Kita-wa sono Kita-Dat opération-Acc demander-Pst Eiziro. Mais Kita-Top cette syuzyutu-o kotowari, [...]. opération-Acc refuser, [...]. 'Eiziro qui, après avoir appris que l'opération de Miyamura est risquée, a voulu en charger Kita, qui travaille dans un service différent. Mais celui-ci a refusé d'effectuer cette opération et [...].' (JQA, 02/05/2003) 

présente une situation comme un bloc sémantique : dans chaque cas, le SN correspond au sujet du SV. Dans [34a], la situation dénotée par la phrase averbale entière est reprise par l'expression sono zizitu 'ce fait'. En second lieu, le transfert du point de vue est mis en œuvre: dans [34a], le prédicat, mitome-taku-nai 'ne pas vouloir admettre', décrit un état psychologique qui n'est compatible avec le sujet de troisième personne que dans un style non rapporté monologique (voir la section 1.2). Mais la déicticité n'y est pas observée : le prédicat mitome-taku-nai 'ne pas vouloir admettre', dans [34a], décrit une situation non perceptible. Dans [34b], dont le SV est mis au passé, la contrainte sur le temps est levée. mitome-taku-nai 'ne pas vouloir admettre' dans [34a] décrit un état, et tanon-da 'demanderPst' dans [34b], un achèvement. Corrélativement, le type de la relation de discours établie avec la phrase suivante est aussi varié : dans [34a], la phrase averbale sert d'Arrière-plan, tandis que dans [34b], elle produit un Contraste, comme l'indique le conjonctif sikasi 'mais'.

Il est à noter que le second type de phrase averbale apparait dans un autre type de texte et à une autre place qu'au début du texte. La phrase averbale dans [35] est tirée d'un article rédigé par un mannequin qui a participé au concours de Miss Monde. Avant le paragraphe cité, l'auteur parle de son grand-père qui a survécu à la bataille d'Okinawa, alors que l'armée japonaise aurait voulu le forcer à se suicider, et de Miss Liban qu'elle a rencontrée à l'occasion du concours et qui éprouvait une vive inquiétude à la suite de l'éclatement de la guerre entre le Liban et Israël :

[35]

[au milieu d'un article sur la situation politique actuelle du Japon]

nihon-no wakamono-wa seezi-ni kansin-ga nai to iwa-re-masu Japon-Gen jeunes-Top politique-Dat intérêt-Nom Neg Comp dire-Pas Poli ga, soosita sinkokuna zyookyoo-o kakaenu koto-mo naku, ikite-ikeru mais une telle grave situation-Acc éprouver fait-aussi sans, vivre-pouvoir karade-wa nai-ka to, kangaekon -desimai- masi-ta. [sv nagai heewa parce que-Top Neg-Q-Comp se demander-finir par-Poli-Pst long paix -no zidai-ga tuzuki keezaitekini -mo megumare-tekita] [sN nihon]. Gen période-Nom durer économiquement-aussi favorisé-avoir été Japon itu-no ma-ni-ka, zibun-no koto-bakari de hito-no itami-ni sans le remarquer, soi-Gen affaire-seulement et autrui-Gen douleur-Dat omoi -ga itara-nai yononaka-ni nat-tesimatta node-wa nai-ka attention-Nom prêter-Neg société à devenir-finir par n'est-ce pas

taikai syutuzyoo-o ki-ni [...] watasi-wa sonna omoi-o sélection participation-Acc depuis $[\ldots]$ moi-Top une telle impression-Acc tunorasetei-masu.

renforcer-Poli

'En entendant dire que les jeunes Japonais ne s'intéressent pas à la politique, je finis (poli) par me demander si c'est parce que nous, les jeunes, pouvons vivre sans être impliqués dans une situation aussi grave [ex. la bataille d'Okinawa pour son grand-père et la guerre entre le Liban et Israël pour Miss Liban]. Le Japon qui a vu durer une longue période de paix et a été favorisé aussi économiquement. Notre société n'est-elle pas devenue imperceptiblement une société où l'on ne pense qu'à soi-même et ne prête pas attention aux douleurs d'autrui ? - Depuis la participation au concours, je ressens (poli) cette impression de plus en plus vivement.' (JQA, 25/08/2009)

Cet exemple montre les particularités du second type. Le SV décrit un état qui dure depuis longtemps et n'est donc pas ancré dans la situation de perception. D'autre part, le transfert énonciatif est clairement observé : les prédicats dans cet article sont en principe 
mis à la forme polie qui indique un style dialogique, tandis que ceux de la phrase averbale et de la question qui la suit sont dépourvus de formes de politesse, ce qui indique que ces deux phrases sont écrites au style non interactionnel. La différence des niveaux énonciatifs est aussi indiquée par le tiret qui marque le retour au style dialogique. Quant à la relation de discours, la question qui suit la phrase averbale sert de Résultat, étant donné la paraphrase possible : «Le Japon qui a vu durer une longue période de paix. Ne résulte-t-il pas de là que notre société est devenue une société où l'on ne pense qu'à soimême?».

Les deux phrases averbales dans [36], exemple tiré du compte rendu d'un livre académique, peuvent être analysées de la même manière. Ici, la première phrase averbale est formée d'une nominalisation et coordonnée à la seconde. Cela suggère que l'une et l'autre présentent la situation comme un bloc sémantique. Le transfert du plan énonciatif est aussi clairement indiqué par les deux questions directes, que l'auteur du compte rendu pose à la place de l'auteur du livre, en s'identifiant à lui :

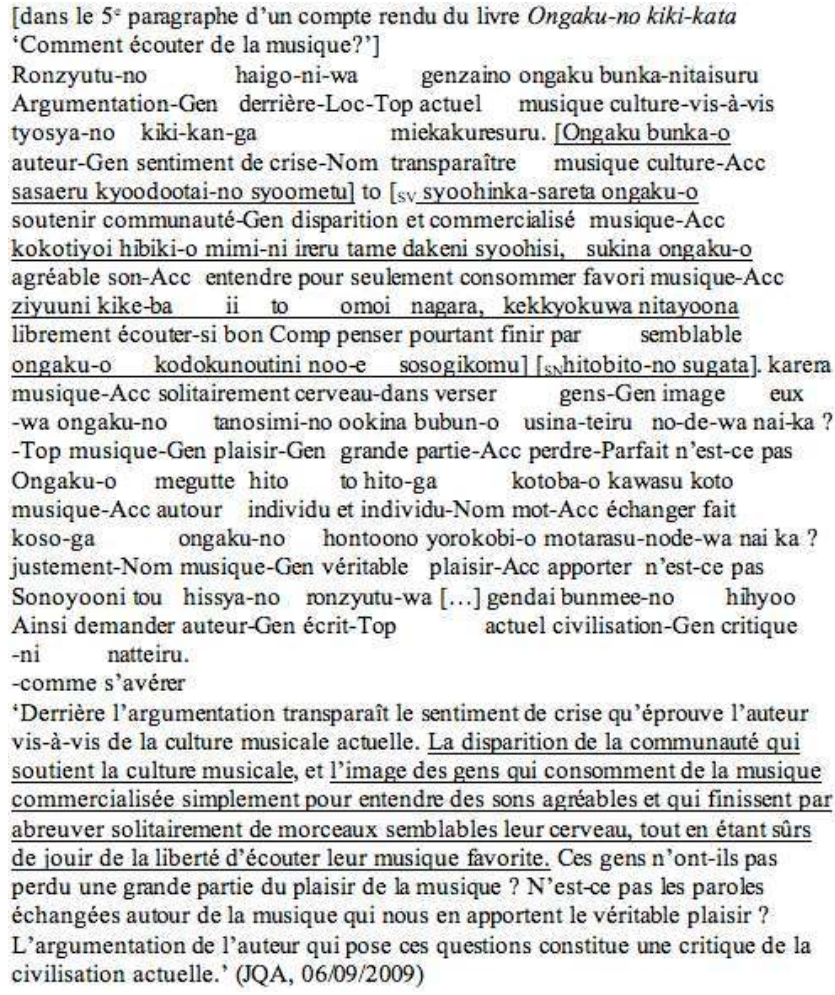

51 Pour la relation de discours, les deux phrases averbales servent d'Élaboration à la phrase précédente : elles décrivent les détails du kikikan 'sentiment de crise' de l'auteur du livre. De plus, l'aspect d'achèvement de syoometu 'disparition', dans la première phrase averbale, permet d'établir une relation de Narration avec la seconde phrase averbale.

\subsection{Type introduisant un topique ou un cadre spatio-temporel sur un nouveau plan énonciatif}

52 Le troisième type de phrase averbale est illustré par [37] et [38], tirés du début du synopsis d'un film. Dans un tel texte, aucune connaissance n'est préalablement partagée 
entre l'auteur et les lecteurs, ni sur le référent du SN, ni sur le procès du SV. L'objectif de ce type est, à la différence du second type, d'introduire un protagoniste, comme dans [37], ou un cadre spatio-temporel dans lequel se développe l'action du film, comme dans [38]. D'autre part, l'information apportée par le SV est à accorder avec les connaissances des lecteurs, c'est-à-dire qu'elle est censée être déjà présente dans leur fonds privé :

[37]

[au début du synopsis du film Le Fabuleux destin d'Amélie Poulain]

[sv Paris-no Montmartre-ni sumi café-de hataraku 22 sai-no]

Paris-Gen Montmartre-Loc habiter café-Loc travailler 22 ans-Gen

[sN Amélie]. osanai-koro-kara utikina kanozyo-wa [...]

Amélie. enfance -depuis timide elle -Top

'Amélie, qui a 22 ans, habite à Montmartre, à Paris, et travaille dans un café.

Cette jeune fille, timide depuis son enfance, $[\ldots] . '(=[4])$

[38]

[au début du synopsis du film Lost in Space]

[sv 2058 nen, kankyoo -hakai-ga susumi sigen-ga

2058 année environnement-destruction-Nom s'aggraver ressource-Nom

nakunat-ta] [sN tikyuu]. zinrui-wa aratana wakusee-e utura-

se tarir-Pst terre espèce humaine-Top nouveau planète-vers déménager-

nakerebanaranakunat-ta.

devoir -Pst

'2058. La terre, sur laquelle la destruction environnementale s'est aggravée et les ressources naturelles se sont taries. L'espèce humaine est obligée de partir pour une nouvelle planète.' (JQA, 05/05/2003)

Ce type de phrase averbale ne satisfait pas deux des trois caractéristiques du Kantai. D'abord, la déicticité n'est pas observée : le prédicat est de type habituel dans [37], et décrit un événement déjà accompli dans [38]. En second lieu, il ne décrit pas une situation en bloc sémantique : dans [38], le SN correspond à un complément circonstanciel du SV. Dans [37], la reprise pronominale ne porte pas sur la situation dénotée, mais seulement sur le référent du $\mathrm{SN}$ qui sert de topique discursif. L'hypothèse suivant laquelle cette construction a pour but d'introduire un topique discursif est confirmée par le fait que le synopsis n'est parfois formé que d'une séquence en $<\mathrm{SV}+\mathrm{SN}>$ accompagnée du marqueur de topique -wa et de points de suspension, comme dans [39] :

[39]

[au début du synopsis du film Pretty Woman]

[sv Fu-annaina nisikaigan-de syakoo-zyoo-no hanryo-ni wakai

Non familier Côte d'ouest-Loc relations sociales-Gen partenaire-pour jeune

call girl-o yatot-ta] [sN zitugyooka]-wa [...].

prostituée-Acc embaucher-Pst homme d'affaires-Top [...].

'Homme d'affaires qui a embauché une jeune prostituée comme partenaire dans les activités de sa société, il [...].' (JQA, 06/09/2009)

Mais ce type de phrase averbale sert à déplacer le plan énonciatif. C'est confirmé dans

[40] par le fait que le texte commence par un commentaire concernant des aspects 
extérieurs du film (un de ses acteurs et son succès commercial), tandis que la phrase averbale guide les lecteurs à l'intérieur de l'action :

[40]

[au début du synospis du film The Terminator]

Arnold Schwarzenegger-ga reekoku-muhina satuzin-machine

Arnold Schwarzenegger-Nom cruel-incomparablement meutre-machine

Terminator-ni hunsi-ta hitto-saku.

Terminator-Dat jouer-Pst succès-cuvre

[sv computer-ni kenryoku-o syooakus-are, zinrui-ga zetumetu

ordinateur-par pouvoir-Acc saisir-Pas, espèce humaine-Nom disparition

-no kiki-ni hinsi-teiru 2029 nen-kara yatteki-ta] [ss Terminator].

-Gen danger-en être-Prog 2029 année-Abl venir-Pst Terminator.

'(C'est) une œuvre qui a connu un grand succès, dans laquelle Arnold

Schwarzenegger joue le rôle d'une machine meurtrière incomparablement cruelle, Terminator.

Terminator qui vient de l'année 2029 où l'espèce humaine est en danger de

disparition, ayant laissé saisir le pouvoir par les ordinateurs.' (JQA, 26/10/2003)

\subsection{Type introduisant un topique discursif sans le transfert du plan énonciatif}

Le quatrième type de phrase averbale est illustré par [19] (voir la section 1.3) et [41a]. Ce type ne présente aucune des trois caractéristiques du Kantai. D'abord, la déicticité n'est pas observée : le référent du SN dans [41a] n'est pas une entité particulière observable, mais une espèce, et le SV ne dénote pas un événement directement perçu. Ensuite, il ne s'agit plus d'un bloc sémantique, ce qui est montré par le fait que ce n'est pas l'ensemble de la construction, mais le $\mathrm{SN}$ seul qui est repris comme topique implicite de la phrase suivante. Enfin, ce type ne met pas en œuvre un transfert du plan énonciatif, ce qui est confirmé par le fait que, si le texte entier est rédigé dans un style interactionnel avec le marqueur de politesse, le SV doit être accompagné de la même marque, comme dans [41b]:

$$
\text { [41a] }
$$

[au début d'un article scientifique sur l'amélioration des espèces de concombres]

[sv Inde-Himalaya-no yama -suso-ga gensan to sa $\quad$-reru] Inde-Himalaya-Gen montagne-pied-Nom origine comme considérer-Pas [sN kyuuri]. Nihon-e-no denrai-wa 10 seeki izen to sa-reru. concombre Japon-dans importation-Top 10 siècle avant Comp considér-Pas 'Les concombres qui sont considérés comme originaires du pied de l'Himalaya en Inde. Ils sont considérés comme ayant été importés avant le $\mathrm{X}^{\circ}$ siècle.' (JQA, 29/08/2009)

[41b] 
[sv Inde-Himalaya-no yama -suso-ga gensan to sa -re

Inde-Himalaya-Gen montagne-pied-Nom origine comme considérer-Pas

-masu] [sN kyuuri]. Nihon-e-no denrai-wa 10 seeki izen to

-Poli concombre Japon-dans importation-Top 10 siècle avant Comp

sare-tei-masu.

considérer-Prog-Poli

'Les concombres qui sont (poli) considérés comme originaires du pied de

l'Himalaya en Inde. Ils sont (poli) considérés comme ayant été importés avant le $\mathrm{X}^{\circ}$ siècle.'

56 L'objectif de ce type n'est que d'introduire le référent du SN comme un topique discursif portant sur le texte entier.

\subsection{Récapitulation}

Cette section a montré, sur la base d'exemples tirés de la presse, qu'il faut reconnaître, pour la phrase averbale en $<\mathrm{SV}+\mathrm{SN}>$ du japonais, non seulement le type du Kantai, mais aussi trois autres types qui sont dépourvus d'une, deux ou trois des caractéristiques de celui-ci. Les résultats de cette section sont récapitulés dans le tableau suivant:

Type 1 : simple réaction cognitive vis-à-vis d'une perception directe

+ déicticité : identifiée par les contraintes sur le prédicat (perceptible et temporaire) et sur le temps

+ bloc sémantique : identifié par la reprise au moyen d'un pronom neutre ou d'un nom dénotant un événement ou une situation, et par la contrainte sur le rôle grammatical du SN

+ transfert du plan énonciatif : identifié par la compatibilité entre le sujet de troisième personne et les prédicats de sensation ou de sentiment, ou bien par la présence du tiret ou des points de suspension

Type $2:$ présentation d'une situation sur un nouveau plan énonciatif

- déicticité

+ bloc sémantique

+ transfert du plan énonciatif

Type 3 : introduction d'un topique discursif ou d'un cadre spatio-temporel sur un nouveau plan énonciatif

- déicticité

- bloc sémantique

+ transfert du plan énonciatif

Type 4 : introduction d'un topique discursif sans le transfert du plan

énonciatif

- déicticité

- bloc sémantique

- transfert du plan énonciatif

\section{Sommaire conclusif}

Cette étude a d'abord passé en revue, dans la section 1 , les travaux antérieurs des linguistes sur la phrase averbale en $<\mathrm{SV}+\mathrm{SN}>\mathrm{du}$ japonais, et a constaté qu'ils ont dirigé leur attention presque exclusivement sur un de ses emplois. Ce type d'emploi, appelé Kantai (Yamada, 1936), transmet une simple réaction vis-à-vis d'une perception directe, et est mis en parallèle avec la pseudo-relative en $<\mathrm{SN}$ qui $\mathrm{SV}>$ du français. Il est caractérisé par trois valeurs (déicticité, présentation d'un événement comme un bloc sémantique, 
transfert du plan énonciatif) dont chacune est par ailleurs identifiée par des critères explicites.

J'ai ensuite observé, dans la section 2, i) que les phrases averbales dans la fiction en français et en anglais servent aussi à déplacer la perspective ou le plan énonciatif, ii) que la fonction discursive d'une phrase averbale dans les didascalies et la presse est solidaire de sa place dans le texte, et iii) que les phrases averbales en nominalisation mettent en œuvre une interaction entre le type aspectuel du procès et le type de relation du discours. Ces observations m'ont conduit, dans la section 3, à distinguer trois autres types de phrase averbale, caractérisés respectivement par le manque d'une, deux ou trois des trois caractéristiques du Kantai. Cela suggère que les trois types sont peut-être dérivés du type de Kantai. Il serait intéressant de vérifier cette hypothèse en effectuant une recherche diachronique.

D'autre part, la portée de cette étude est limitée à l'analyse des exemples de la presse. Les études ultérieures devront se demander, à l'instar des travaux antérieurs sur les phrases averbales du français, si la même analyse est valable dans les autres genres d'écrits (fiction, didascalie, etc.) ainsi qu'à l'oral ${ }^{19}$. L'étude des phrases averbales à l'oral est d'autant plus intéressante qu'on les entend fréquemment aujourd'hui dans les émissions sportives, comme dans [1b], ainsi que dans les journaux télévisés, comme dans [42] :

[42]

[journal télévisé de 19 heures]

[sv Saranaru kansen kakudai-ga sinpaisa-reru] [ss infuruenza].

Encore plus contagion croissance-Nom inquiéter-Spontané grippe

'La grippe dont la contagion croissante nous inquiète.' (NHK, 06/09/2009)

Je remercie les deux rapporteurs pour leurs remarques précieuses et constructives. Je suis aussi reconnaissant à mon collègue d'Okayama, Michel de Boissieu, pour la correction du français. Toutes les insuffisances qui restent sont dues à l'auteur. Cette étude a reçu le soutien financier de la Japan Society for the Promotion of Sciences (numéro 19520346).

\section{BIBLIOGRAPHIE}

ASHER, N., LASCARIDES, A. 2003. Logic of Conversation. Cambridge : Cambridge University Press.

Beyssade, C., MARAndin, J.-M. 2006. The Speech Act Assignment Problem Revisited : Disentangling Speaker's Commitment from Speaker's Call on Adressee. In O. BONAMI, P. CABREDO HOFHERR (eds), Empirical Issues in Syntax and Semantics $6: 37-68$. Accessible en ligne sur http://www.cssp.cnrs.fr/ eiss6.

CADIOT, P. 1976. Relatives et infinitives « déictiques » en français. DRLAV $13: 1-64$. DELORME, B. 2004. Prédication averbale et ruptures de repérage. Verbum : La phrase averbale : délimitations et caractéristiques XXVI (3). F. Lefeuvre (ed.) : 343-353. 
DELORME, B., LEFEUVRE, F. 2004. De la prédication seconde à la prédication autonome. Verbum XXVI (3) : 281-297.

ERTESCHIK-SHIR, N. 1997. The Dynamics of Focus Structure. Cambridge : Cambridge University Press. FARKAS, D., SWART, H. 2003. The semantics of incorporation. Stanford : CSLI.

FURUKAWA, N. 1996. Grammaire de la prédication seconde. Louvain-la-Neuve : Duculot.

GUILLEMIN-FLESCHER, J. 2004. Les énoncés averbaux : de l'identification à l'évaluation. Syntaxe et Sémantique : Aux Marges de la prédication 6. I. BEHR, J. FRANÇOIS, A. LACHERET, F. LEFEUVRE (eds.). Caen : Presses universitaires de Caen : 132-162.

INOUE, K. 1976. Henkee-bunpoo to nihon-go (ge) (La grammaire transformationnelle et le japonais, vol. 2). Tokyo : Taisyuukan Pub.

KRAZEN, M. 2004. La phrase averbale dans les didascalies. Verbum XXVI (3) : 355-372.

KURODA, S.-Y. 1979. Où l'épistémologie, la grammaire et le style se rencontrent : examen d'un exemple japonais. In S.-Y. KURODA (ed.), Aux Quatre Coins de la Linguistique. Paris : Seuil : 235-259.

KURODA, S.-Y. 1992. Judgment Forms and Sentence Forms. In S.-Y. KURODA (ed.), Japanese Syntax and Semantics. Dordrecht : Kluwer : 13-77.

LÉARD, J.-M. 1992. Les gallicismes. Louvain-la-Neuve : Duculot.

LEFEUVRE, F. 1999. La Phrase averbale en français. Paris : l'Harmattan.

LEFEUVRE, F. 2004a. Le prédicat nominal dans les articles de presse. Syntaxe et Sémantique 6 :

181-198.

LEFEUVRE, F. 2004b. La temporalité des nominalisations prédicatives. Verbum XXVI (3) : 311-326.

MARANDIN, J.-M. 2005. Formatage de l'information : focus et contexte. In F. CORBLIN, C. GARDENT (eds), Interpréter en contexte. Paris : Lavoisier : 31-79.

MiKAMI, A. 1972. Zoku gendai gohoo-zyosetu (Suite de l'introduction de l'usage moderne du japonais). Tokyo : Kurosio Pub.

ONOE, K. 2001. Bunpoo to imi I (Grammaire et sens I). Tokyo : Kurosio Pub.

SANDFELD, K. 1965. Syntaxe du français contemporain 2 : les propositions subordonnées. Genève : Droz. SASSE, H.-J. 1987. The Thetic / Categorical Distinction Revisited. Linguistics 25 : 511-580.

TERAMURA, H. 1993, Teramura Hideo ronbun-syuu I (Recueils des articles de Hideo Teramura I). Tokyo : Kurosio Pub.

Тsuвомото, А. 1992. Teezibun to gensyoo-bun (Phrases présentatives et jugement thétique). Bunka-gengogaku (Linguistique culturelle). Tokyo : Sanseedoo Pub. : 564-578.

Tsuвомото, А. 2000. Bare subject inversion construction in Japanese. In K. TAKAMIet al. (eds), Syntactic and Functional Explorations. Tokyo : Kurosio Pub.

YAMADA, Y. 1936. Nihon-bunpoo-gairon (Traité de grammaire du japonais). Houbunkan Pub.

\section{NOTES}

1. Les abréviations utilisées dans cette étude sont les suivantes : $A b l=$ ablatif $; A c c=$ accusatif ; Caus $=$ causatif $;$ Comp $=$ complémenteur $;$ Cop = copule $; \mathrm{Gen}=$ génitif $;$ Loc = locatif $;$ Nom = 
nominatif $;$ Pas $=$ Passif $;$ Prog $=$ progressif $; \mathrm{Neg}=$ négation $;$ Pst $=$ passé $;$ Top $=$ topique $; \mathrm{SN}=$ syntagme nominal; $\mathrm{SV}=$ syntagme verbal.

2. En ancien japonais, les verbes manifestent la distinction entre les formes conclusive et adnominale. Voir la section 1.1 .

3. «Seko » est un coureur de marathon.

4. «hasiru»: le japonais est dépourvu de concordance des temps et le prédicat subordonné concomitant à celui de la principale reste morphologiquement non marqué.

5. «Nakamura Syunsuke » est un joueur de football.

6. Les différences entre la pseudo-relative et les relatives restrictives ou appositives seront discutées dans la section 1.

7. Mikami (1972: 109-112), mentionnant la distinction entre le Kantai et le Zyuttai, critique l'identification de la distinction entre la phrase à topique et phrase sans topique avec celle entre le jugement catégorique (double) et le jugement thétique (simple).

8. La discussion de Cadiot (1976) porte toutefois principalement sur la pseudo-relative introduite par un verbe de perception, comme dans je vois Marie qui pleure.

9. "The distinction between sentences with stage topics [i.e. spatio-temporel topic] and sentences with individual topics is equivalent to the thetic-categorical distinction » (ErteschikShir, $1997: 241$, note 27 ).

10. La phrase nominale du type Kantai a des points communs avec la construction adnominale, comme dans [I], dont la tête dénote une sensation perceptive (ex. visuelle, auditive, olfactive, etc.) :

[I] [ame-ga hagesiku huru] oto (Inoue, 1976:197)

pluie-Nom à verse tomber bruit

'le bruit de la pluie qui tombe à verse'

Dans la seconde construction, le SV dénote un événement spatio-temporellement délimité et n'admet que le présent. Inoue (1976:192) note de plus qu'elle «transmet directement la perception du sujet ». Teramura (1993 : 168) fait remarquer qu'elle « permet à l'interlocuteur de voir, entendre, sentir, etc. au même titre que le locuteur ».

11. La phrase averbale admet l'imparfait dans [I]. Celui-ci semble déplacer le centre déictique sur le passé. Ce problème ne sera pas discuté dans la présente étude dont l'intérêt principal porte sur la phrase averbale japonaise.

[I] Et Marie qui pleurait ! (exemple fourni par un des deux rapporteurs)

12. On peut se demander si le bloc sémantique est équivalent à l'incorporation sémantique (voir Farkas et Swart, 2003, entre autres). On observe certes des cas de la phrase averbale du japonais qui satisfont trois critères de l'incorporation sémantique : i) non-reprise du SN dans le texte ultérieur, comme dans [12b] ; ii) portée étroite par rapport à un quantifieur dans la même phrase. Ainsi, le SN patorooru-kaa 'voiture de police' dans [I] est interprété sous la portée de l'adverbe quantificationnel, hikkirinasini 'sans cesse' ; iii) neutralisation de la distinction singulier / pluriel. Le SN patorooru-kaa dans [I] est ainsi interprété, malgré sa forme nue, comme dénotant la pluralité :

[I] [hikkirinasini ikikisuru] patorooru-kaa. (JQA, 19/11/1997)

sans cesse aller et venir voiture de police

'Des voitures de police qui vont et viennent sans cesse.'

D'autre part, l'incorporation sémantique porte en principe sur un nom commun morphologiquement non marqué, tandis que le bloc sémantique peut concerner même un nom propre, comme dans [12b], qui n'est certes pas repris dans le contexte ultérieur, mais qui n'a pas de portée étroite et ne dénote qu'une entité singulière. L'extension du bloc sémantique est donc plus large que celle de l'incorporation sémantique.

13. Le SN de la pseudo-relative du français manifeste également un des symptômes de l'incorporation sémantique, la portée étroite par rapport à un quantifieur dans la relative. Le SN 
les soldats dans [I] est ainsi sémantiquement intégré au prédicat subordonné se faire tuer dont l'ensemble est interprété sous la portée de l'opérateur itératif continuer :

[I] [Le Président a déclaré que la guerre était finie en Irak]

Et les soldats qui continuent à se faire tuer!

= Il se produit sans arrêt des événements qui entraînent la mort d'un soldat au moins.

14. Le SN accompagné de la relative restrictive ou appositive peut être repris par un pronom neutre dans les phrases génériques, comme dans [I] dont le SV est de type permanent. Il faut donc recourir à la reprise par un nom dénotant une situation dans les cas où le présent du SV est ambigu et autorise à la fois la lecture déictique et la lecture générique :

[I] Une fille qui a les cheveux courts, \{ça / *ette situation\} me plaît toujours.

15. Akebono et Takanohana sont des lutteurs de sumo.

16. La présentation qui suit recourt aux explications et aux exemples d'Asher et Lascarides (2003: 460-463).

17. Cette liste n'est pas exhaustive, et ne mentionne que les types pertinents pour l'analyse de la phrase averbale.

18. Les cris et bousculade pourront être classés, en raison de leur nature itérative, parmi les semelfactifs. Compte tenu du fait que les activités ont aussi des parties non homogènes (ex. nager est classifié parmi les activités, mais quand on nage, tantôt on bouge les mains et les jambes, et tantôt on flotte tout simplement), j'analyserai les semel-factifs comme un type particulier des activités ayant des parties hétérogènes.

19. Il sera aussi intéressant de constituer un corpus franco-japonais, ce qui rendrait possible de déterminer si les 4 types proposés s'appliquent tous aux deux langues de la même façon.

\section{RÉSUMÉS}

Cette étude a pour but d'examiner les diverses fonctions discursives remplies en japonais par une phrase averbale du type < proposition subordonnée + syntagme nominal >. Les travaux antérieurs ont caractérisé cette construction au moyen de trois valeurs: i) expression d'un événement directement perçu comme formant un bloc sémantique, sans la dichotomie entre sujet et prédicat; ii) ancrage de l'événement dans le moment de la perception; iii) transfert du plan énonciatif. Dans cette optique, elle a été mise en parallèle avec une phrase averbale du type « Le lavabo qui déborde!» en français. En se basant sur des exemples tirés de la presse, et en profitant des analyses antérieures sur la phrase averbale dans d'autres langues, la présente étude soutient qu'il faut distinguer, à côté du premier type déictique, trois autres types : i) celui qui présente une situation comme formant un bloc sémantique et introduit un nouveau plan énonciatif, mais n'effectue pas l'ancrage dans le moment de la perception. Ce type établit, avec les phrases précédentes ou suivantes, diverses relations de discours, Arrière-plan, Élaboration ou Contraste ; ii) celui qui introduit un plan énonciatif déplacé, mais ne met pas en relief la situation dénotée, et fait du référent du syntagme nominal un topique discursif ou un cadre spatiotemporel ; iii) celui qui ne présente aucune caractéristique du premier type et se trouve réservé à la mise en relief d'un topique discursif portant sur le texte entier.

This study aims at examining various discourse functions of the Japanese averbal sentence formed by the sequence < subordinate proposition + noun phrase >. Previous studies have characterized this construction in terms of three aspects : i) expression of a directly perceived 
event as forming a semantic unit with no distinction between subject and predicate; ii) anchoring of the event in the moment of perception ; iii) introduction of a new utterance level. From this perspective, the Japanese averbal sentence has been compared with a type of averbal sentence in French, like "Le lavabo qui déborde!". On the other hand, based on examples taken from newspapers, and former works about averbal sentences in other languages, this study claims that, besides the first type extensively discussed, other three types should be distinguished : i) one which expresses a situation as forming a semantic unit and introduces a displaced utterance level, but with no anchoring in the utterance moment. This type establishes various discourse relations, like Background, Elaboration, Contrast with surrounding sentences ; ii) one which introduces a new utterance level, but does not focus on any unitary event, but to make a prominent topic or a spatial or temporal frame of the referent of the noun phrase ; iii) one which shares no characteristics of the first type and is specialized in focusing on a discourse topic whose scope amounts to the whole text.

INDEX

Keywords : anchoring in the situation of perception, averbal sentence, discourse relations, introduction of a new utterance level, Japanese, thetic judgment

Mots-clés : ancrage dans la situation de la perception, japonais, jugement thétique, phrase averbale, relations de discours, transfert du plan énonciatif

\section{AUTEUR}

MAKOTO KANEKO

Université d'Okayama 